\title{
Qualidade de vida e auto-imagem de pacientes com câncer de cabeça e pescoço
}

\section{Quality of life and self-image in patients with head and neck cancer}

\author{
Recibido: junio 15 de 2009 | Revisado: junio 20 de 2010 | Aceptado: noviembre 14 de 2010 \\ Marina Silveira E Silva* \\ ELISA KERN DE CASTRO \\ Universidade do Vale do Rio dos Sinos, \\ São Leopoldo, Brasil \\ Carolina Chem ** \\ Hospital Santa Rita, Complexo Hospitalar Santa Casa \\ de Misericórdia de Porto Alegre, Brasil
}

SICI: 2011-2777(201203)11:1<13:QDVEAI>2.0.CO;2-4

Para citar este artículo: Silveira e Silva, M., Kern de Castro, E. \& Chem, C. (2012). Qualidade de vida e auto-imagem de pacientes com câncer de cabeça e pescoço. Universitas Psychologica, 11(1), 13-23.

* Programa de Pós-Graduação da Universidade do Vale do Rio dos Sinos (UNISINOS), Av. Unisinos 950, Bairro Cristo Rei, São Leopoldo, RS, Brasil. E-mails: emarinass@yahoo.com, elisa.kerndecastro@gmail.com

** Pc D Feliciano, 135. Centro Porto Alegre, RS, 90020-160. E-mail: carolinachem@hotmail.com
R E S U M O

O câncer de cabeça e pescoço refere-se a tumores malignos de pele que atinge pessoas de todas as idades. $O$ tratamento envolve cirurgias, quimioterapia e radioterapia. Objetivo: examinar a auto-imagem e a qualidade de vida de pacientes que tiveram câncer de cabeça e pescoço. Participantes: 28 pacientes, entre 30 e 73 anos. Instrumentos: Avaliação da Qualidade de Vida da OMS - versão breve e Escala de Avaliação com a Satisfação Corporal. Resultados: qualidade de vida total e suas subescalas correlacionaram-se positivamente com auto-imagem, com exceção da subescala relações sociais. A auto-imagem foi uma variável preditora significativa da qualidade de vida total e das dimensões física, psicológica e meio-ambiente. Conclusão: a qualidade de vida desses pacientes está intimamente ligada à auto-imagem. Palavras-chave autores:

qualidade de vida, auto-imagem, câncer de cabeça e pescoço.

Palavras-chave plus:

Psico-oncologia, câncer, bem-estar.

\section{A B S T R A C T}

Head and neck cancer refers to skin tumors that affect people of all ages. Treatments for this type of disease include surgeries, chemotherapy and radiotherapy. Purpose: to examine self-image and quality of life of patients who had head and neck cancer. Participants: 28 patients between $30-73$ years. Instruments: Instrument for Assessing Quality of Life of the WHO - and the brief version of Evaluation Scale with Body Satisfaction. Results: Total quality of life and their subscales was positively correlated with selfimage, with the exception of social relations subscale. Self-image was a significant predictor of total quality of life and physical psychological and environment dimension. Conclusion: quality of life was closely linked with self-image in these patients.

Key words authors:

Quality of Life, self-image, head and neck cancer.

Key words plus:

Psycho-oncology, cancer, well-being. 


\section{Introdução}

Entre as neoplasias humanas malignas a mais comum é o câncer de pele, sendo a região da cabeça e pescoço a área mais freqüentemente envolvida $90 \%$ entre os homens e $85 \%$ entre as mulheres. Sua incidência tem aumentado durante as últimas três décadas (Instituto Nacional do Câncer [INCA], 2010; Kowalski, 2005; Nora, Panarotto, Lovatto $\&$ Boniatti, 2004). As pessoas acometidas desse tipo de tumor podem ficar com a face desfigurada, sendo que em alguns casos sem partes inteiras do rosto. Geralmente o tratamento é à base de cirurgia para a retirada do tumor, cirurgia reconstrutiva, quimioterapia e radioterapia.

Os tumores malignos da pele mais comuns são: carcinoma basocelular (60\%), o carcinoma espinocelular (30\%) e o melanoma (cerca de $6 \%$ ), sendo os $4 \%$ restantes de histologia variada (Sociedade Brasileira de Dermatologia, 2006; INCA, 2010). Didaticamente é possível dividir os tumores cutâneos malignos em dois grandes grupos: não-melanoma (carcinomas basocelular e epidermóide) e melanoma.

Dentre os fatores de risco para câncer de pele não-melanoma estão: a exposição às radiações ultravioleta, a exposição a agentes químicos, a presença de papiloma vírus humano, as radiações ionizantes e a imunossupressão. Por ocasião do diagnóstico, um quarto dos pacientes apresenta mais de uma lesão, e aproximadamente metade deles tem acima de sessenta anos. Esse tipo de câncer se caracteriza pela baixa letalidade, porém deixa marcas profundas, como deformidades físicas e ulcerações graves (INCA, 2010). Já o melanoma é uma neoplasia originária das células responsáveis pela pigmentação cutânea e é a principal causa de morte entre as doenças de pele, apesar de sua incidência ser de apenas 4 \% (Carvalho, Cunha, Gigliani, Bakos \& Ashton-Prolla, 2004; Vidrio \& Lozano, 2007). Entre os fatores de risco para o melanoma estão: presença de numerosos nevos, nevos atípicos, cabelo ruivo, pele clara e história familiar de melanoma.

A palavra câncer ainda é tida como uma sentença de morte prévia (Carvalho, 1998). Dor, so- frimento físico e psicológico, e perda da qualidade de vida são situações comumente associadas para quem recebe o diagnóstico. Atualmente, com os avanços da medicina, a sobrevida dos pacientes com câncer aumentou substancialmente, e por isso se torna importante estudar a qualidade de vida dessas pessoas, uma vez que sobrevida não implica em qualidade de vida.

No Brasil, há escassos estudos sobre os aspectos psicológicos do câncer de cabeça e pescoço, apesar de haver equipes especializadas no atendimento desses pacientes. De acordo com Rogers, Ahad e Murphy (2007), Rumsey, Clarcke, White, Wyn-Williams e Garlick (2004) e Katz (2000), na última década percebe-se na literatura internacional um aumento do reconhecimento da importância da mensuração da qualidade de vida em pacientes com câncer de cabeça e pescoço. Esse aspecto é importante não apenas para descrever as questões inerentes a essa população, mas também para discutir possíveis intervenções.

O termo qualidade de vida foi introduzido na área da saúde devido ao avanço tecnológico que, além de novas reabilitações, proporcionou um aumento na expectativa de vida de pacientes crônicos (Diniz \& Schor, 2006; Seidl \& Zannon, 2004). A mudança de visão sobre o ser humano, em que ele passa a ser visto como um todo considerando seus aspectos biopsicossociais, fez com que a preocupação com a saúde integral das pessoas passasse a ser considerada. $O$ interesse pela qualidade de vida em amostras clínicas e a aplicação de instrumentos específicos para avaliá-la são cada vez mais utilizados, incluindo aspectos de sua qualidade de vida objetiva e subjetiva (Remor, 2005).

Em meados dos anos 70, o termo qualidade de vida passou a ser empregado na área da saúde visando o bem-estar do paciente oncológico (Diniz \& Schor, 2006). Pensar em qualidade de vida com esses pacientes é fundamental, pois se trata de uma doença extremamente agressiva e mutiladora, que gera sofrimento tanto para o paciente quanto para sua família (Seidl \& Zannon, 2004).

No ano de 1994, a OMS (WHOQOL Group) padronizou pela primeira vez o conceito de qualidade de vida, incluindo os aspectos subjetivos, de 
unificação do conceito e os aspectos transculturais. Qualidade de vida foi definida tanto como uma percepção individual do sujeito sobre sua saúde (culturalmente, em termos de relacionamentos e com seus valores morais e éticos), como com relação aos seus objetivos, expectativas, padrões e preocupações. Esse é um conceito amplo, influenciado pela saúde física, estado psicológico, nível de independência, relações sociais e da relação com características do ambiente (Diniz \& Schor, 2006; World Health Organization [WHO], 2010).

A auto-imagem, por outro lado, refere-se a uma representação cognitiva e emocional do corpo que é aprendida pelo indivíduo (Price, 1990; Schilder, 1999). Seu conceito define como nos sentimos em relação à nossa aparência física e ao nosso corpo através de uma idéia de multidimensionalidade. Esse conceito vai ser formando a partir da realidade corporal, de como o corpo se apresenta e do ideal de corpo (Schilder, 1999).

Segundo Schilder (1999) e MacGregor (1990), o contato com o outro, na convivência social, vai formando a nossa noção de imagem do corpo. $\mathrm{O}$ olhar se torna fator fundamental e estruturante na nossa relação com o mundo e, consequentemente, na nossa auto-imagem. A face, em especial, simboliza o sujeito e o diferencia dos demais, sendo o foco de atenção na interação com o outro.

Segundo Price (1990), quando ocorre algum tipo de alteração na auto-imagem, o sujeito definirá a nova imagem que tem de si a partir das suas experiências. Os sentimentos e as atitudes relacionadas à imagem corporal formam um conceito de corpo importante para a qualidade de vida, pois a imagem corporal é um aspecto central na auto-estima do sujeito.

As conseqüências psicológicas relacionadas à incapacidade física e perda de alguma parte do corpo refletem em alterações na imagem corporal e manifestam-se no dia-a-dia das pessoas afetadas (Harris \& Carr, 2001; Oliveira, 2004). Independente da maior ou menor gravidade da situação em que o sujeito se encontra, as respostas emocionais vão variar de maneira singular. A auto-imagem está relacionada à insatisfação, distorção, depreciação e preocupação com o seu corpo, sendo todos esses itens influenciados por questões sócio-culturais, fazendo com que essa experiência emocional seja variada. Alguns sintomas podem ser comumente observados em pessoas com perdas e/ou mudanças importantes no corpo: dificuldade nos relacionamentos íntimos e interpessoais, diminuição de relações sociais, sentimentos de isolamento e de constrangimento.

De acordo com Pedrolo e Zago (2000) e Schilder (1999) pode-se dizer que sempre haverá algum grau de alteração na imagem corporal quando ocorrer lesões em alguma parte do corpo. A cirurgia por si só já traz alterações na pele com o corte e sua cicatriz. Porém, alguns tipos de cirurgia, bem como a localização onde esta é feita, podem acarretar danos agressivos à auto-imagem do sujeito. Inclui-se nessas situações pacientes que necessitam de algum tipo de intervenção na face, pois segundo Shilder (1999) esta é a principal parte do corpo que está em comunicação com o outro.

Segundo Vickery, Latchford, Hewison, Bellew e Feber (2003), Katz, Irish, Devins, Rodin e Gullane (2003), Newell (1999) e Moss (2005), pessoas que sofreram algum tipo de desfiguramento em razão de câncer na face vivenciam essa experiência como traumática, acarretando danos psicológicos a esses sujeitos. Ocorrem também alterações na auto-imagem e na qualidade de vida, levando ao impacto negativo na auto-estima.

Cicatrizes e alterações severas na região facial, mais do que em outras regiões, são fatores que podem desencadear graves problemas psicológicos e alterações significativas na auto-imagem do sujeito (Amar, Rapoport, Franzi, Bisordi \& Lehn, 2002; Callanhan, 2004; Gilony et al., 2005). Moss (2005), Bull e Rumsey (1998) e Newell e Clarke (2000), relatam que as alterações na convivência social de pacientes com câncer na face são bastante comuns e trazem à tona a importância dos aspectos psicossociais na vida desses pacientes, pois a maior fonte de sofrimento é oriunda do embaraço sofrido nas situações sociais.

Portanto, parece que os avanços da área médica ainda precisam ser acompanhados pela Psicologia. A falta de estudos nessa área demonstra a impor- 
tância de se investigar questões relacionadas à qualidade de vida do paciente com câncer de cabeça e pescoço. Dessa forma, o presente trabalho buscou examinar a qualidade de vida e a auto-imagem de pacientes com câncer na face, em função da grave mutilação sofrida na região do rosto. Além disso, pretendeu identificar a influência da auto-imagem na qualidade de vida de pacientes que tiveram câncer de cabeça e pescoço.

\section{Método}

\section{Participantes}

Participaram desse estudo 28 pacientes com neoplasias malignas de pele no rosto (não-melanoma), com necessidade ou não de reconstrução na face.
Todos eles eram adultos entre 30 e 73 anos de idade, e estavam em tratamento em um hospital referência em câncer na cidade Porto Alegre. Os pacientes eram tanto do pré-operatório quanto do pós-operatório. A seleção da amostra foi por conveniência: nos dias de consultas de revisão desses pacientes, a pesquisadora que coletou os dados convidou para participar da pesquisa todos aqueles pacientes que estavam aguardando a consulta e tinham tempo disponível para preencher os questionários. Foi critério de inclusão na pesquisa que os pacientes fossem adultos (igual ou maior de 18 anos). Foram excluídos da amostra aqueles que tinham feito laringectomia e, portanto, não se comunicavam verbalmente. A tabela 1 mostra as principais características dos sujeitos

\section{TABELA 1}

Dados sócio-demográficos e clínicos

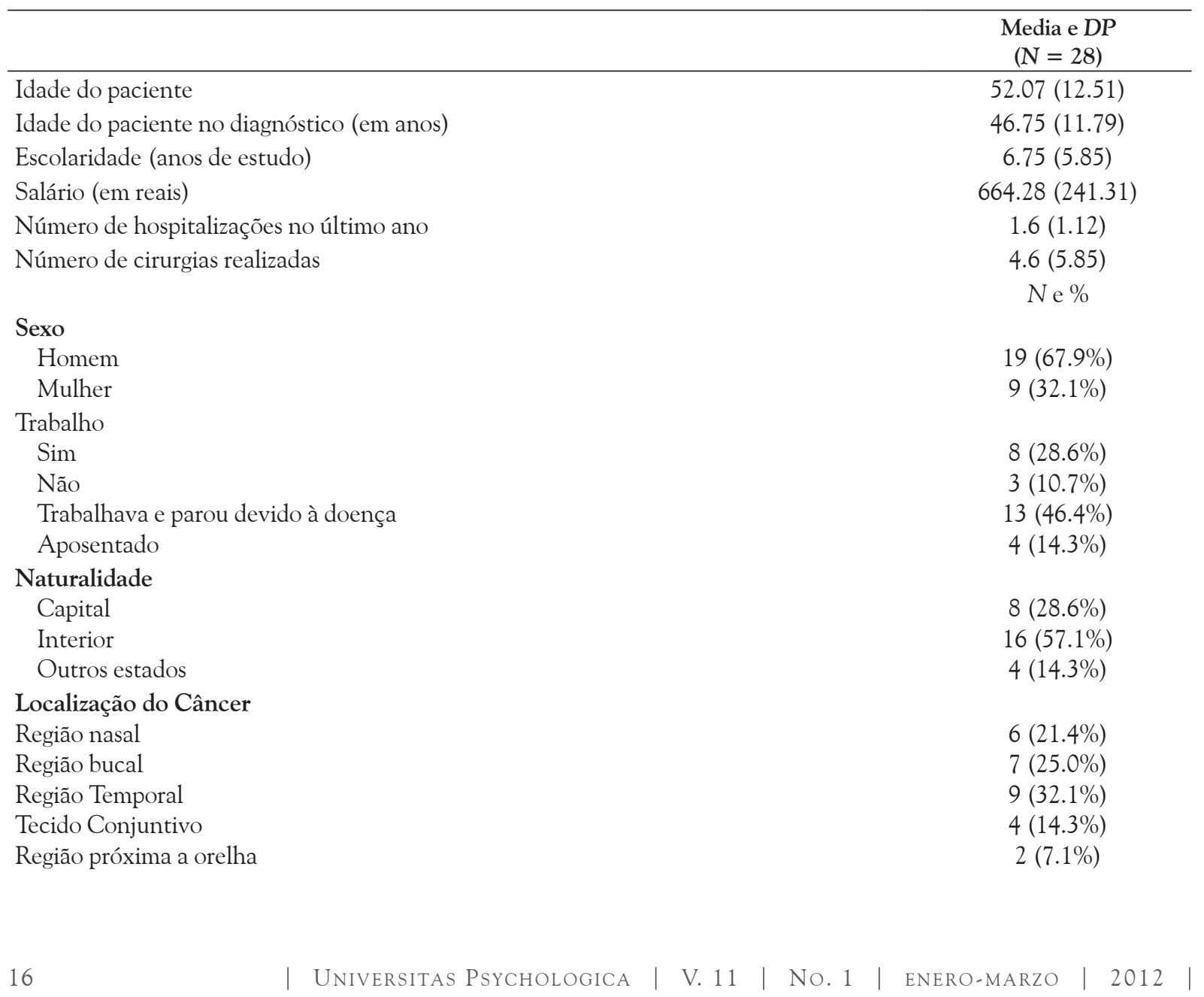




\begin{tabular}{lc}
\hline Tipo de tratamento & $\begin{array}{c}\text { Media e DP } \\
(\mathrm{N}=28)\end{array}$ \\
Quimioterapia & $2(7.1 \%)$ \\
Cirurgia e Quimioterapia & $10(35.7 \%)$ \\
Cirurgia & $8(28.6 \%)$ \\
Cirurgia e Radioterapia & $2(7.1 \%)$ \\
Cirurgia, Radioterapia e Quimioterapia & $3(10.7 \%)$ \\
Ainda não realizou tratamento & $3(10.7 \%)$ \\
Histórico Familiar de câncer & \\
Não & $13(46.4 \%)$ \\
Sim, um dos pais tiveram algum tipo de câncer & $7(25.0 \%)$ \\
Sim, um dos pais tiveram câncer de pele & $8(28.6 \%)$ \\
Psicofármacos & \\
Sim & $16(57.1 \%)$ \\
Não & $12(42.9 \%)$ \\
\hline
\end{tabular}

Fuente: elaboración propia.

\section{Delineamento e procedimento da pesquisa}

Trata-se de uma pesquisa transversal e correlacional. Após aceitação da equipe de Câncer de Cabeça e Pescoço e da psicóloga do hospital em colaborar com o estudo, o projeto foi submetido e aprovado pelo comitê de ética do Complexo Hospitalar Santa Casa de Porto Alegre. A coleta de dados foi realizada no próprio hospital em uma sala do ambulatório do Sistema Único de Saúde, onde o paciente passava depois de sua consulta médica. Todos os participantes assinaram o Termo de Consentimento Livre e Esclarecido. As aplicações dos instrumentos foram individuais.

\section{Instrumentos}

\section{Dados bio-socio-demográficos e clínicos}

Instrumento elaborado para fins do presente estudo, abordava informações importantes sobre o paciente tais como idade, trabalho, estado civil, e dados sobre seu problema de saúde e tratamento.

Instrumento de Avaliação da Qualidade de Vida da Organização Mundial da Saúde. Versão breve (WHOQol-breve) (Fleck, Louzada, Xavier, Chachamovich, Vieira, Santos \& Pinzon, 2000)

É um instrumento desenvolvido pela OMS que objetiva avaliar a qualidade de vida em diferentes culturas. A versão breve do questionário contém 26 itens e deriva da versão original de 100 itens (Fleck et al., 1999). As versões em português/brasileiro de ambos os instrumentos foram desenvolvidas no Departamento de Psiquiatria e Medicina Legal da UFRGS. O WHOQol-breve consta de duas questões gerais e de 24 questões que compõem cada uma das 24 facetas que compõem o instrumento original. Os quatro domínios do instrumento são: físico, psicológico, relações sociais e meio-ambiente. A qualidade de vida total refere-se à soma das quatro dimensões mencionadas. Para cada questão existem 5 graus de intensidade e o paciente escolhe uma delas. Assim, a pontuação mínima que uma pessoa pode obter é 26, e a máxima é 130 pontos. Quanto à consistência interna da qualidade de vida geral, o valor alpha de Cronbach encontrado para o presente estudo foi 0.73, que é aceitável, porém inferior ao encontrado no estudo de validação do instrumento para a população brasileira, que foi de 0.91 (Fleck et al., 2000).

\section{Escala de Avaliação com a Satisfação Corporal}

É um instrumento desenvolvido por Ferreira e Leite (2002) com o objetivo de avaliar a satisfação com a imagem corporal para amostras brasileiras. É composto por vinte e cinco itens que se dividem em duas sub-escalas: Satisfação com a aparência e 
Preocupação com o peso. Tendo em vista que os pacientes da presente pesquisa apresentavam diagnóstico de câncer e o interesse é a imagem corporal relacionada à desfiguração da face, foi utilizada apenas a escala de satisfação com a aparência. Essa escala consta de 18 perguntas e, para cada questão existem 5 graus de intensidade que variam desde "discordo totalmente" a "concordo totalmente" e o paciente escolhe uma delas. Quanto maior a pessoa pontua na escala, melhor é a satisfação com a sua imagem corporal. Verificou-se alpha de Cronbach de 0.61 no presente estudo, um pouco inferior ao do estudo de Ferreira e Leite (2002) que foi de 0.73.

\section{Resultados}

Inicialmente, foi realizado o teste de Shapiro-Wilk e de Kolmogorov-Smirnov para examinar a normalidade da amostra, utilizando o software SPSS 16.0. Ambos os testes mostraram distribuição normal da amostra. A tabela 2 mostra os principais dados descritivos da amostra com respeito à qualidade de vida e auto-imagem.

No intuito de examinar os escores de qualidade de vida e auto-imagem desses pacientes advindos da classificação de baixa, média ou alta QV e auto-imagem, obteve-se a classificação descrita na Tabela 3. É importante ressaltar que, como a qualidade de vida é um conceito subjetivo, não há menção ao estabelecimento de pontos de corte para a população brasileira ou para outras populações (Fleck et al., 2000). Assim, para a classificação do nível de qualidade de vida dos pacientes deste estudo, utilizou-se os quartis apresentados na Tabela 2. Observa-se que a maioria dos pacientes apresentava qualidade de vida total alta, qualidade de vida física, psicológica e ambiental média, porém a qualidade de vida na dimensão relações sociais foi a que se mostrou mais prejudicada para a maioria.

\section{TABELA 2}

Média, desvio padrão, mediana, quartis e mínimo e máximo das variáveis qualidade de vida e suas dimensões, e auto-imagem

\begin{tabular}{|c|c|c|c|c|c|c|}
\hline Instrumento & Dimensão & Média (DP) & Mediana & 1\% Quartil & 3 Quartil & Min/Max \\
\hline & Total & $\begin{array}{l}81.79 \\
(9.04)\end{array}$ & 80 & 74.5 & 88.5 & $68-99$ \\
\hline \multirow{4}{*}{ Qualidade de vida } & Física & $\begin{array}{l}16.86 \\
(2.52)\end{array}$ & 16.5 & 15 & 19 & $12-22$ \\
\hline & Psicológica & $\begin{array}{l}19.64 \\
(1.89)\end{array}$ & 20 & 18 & 20.7 & $16-23$ \\
\hline & Relações sociais & $\begin{array}{c}10.5 \\
(1.57)\end{array}$ & 10.5 & 10 & 11.7 & $7-14$ \\
\hline & Ambiente & $\begin{array}{l}24.6 \\
(5.4)\end{array}$ & 23 & 21 & 28.7 & $15-35$ \\
\hline Auto-imagem & Satisfação com aparência & $\begin{array}{l}51.86 \\
(5.95)\end{array}$ & 51.5 & 48 & 55 & $41-65$ \\
\hline
\end{tabular}

Fuente: elaboración propia. 
TABELA 3

Classificação dos níveis de qualidade de vida e auto-imagem dos pacientes com câncer de cabeça e pescoço

\begin{tabular}{|c|c|c|c|}
\hline \multirow{16}{*}{ Qualidade de vida } & & & $\%(N)$ \\
\hline & \multirow{3}{*}{ QV total } & Baixo & $25(7)$ \\
\hline & & Médio & $28.6(8)$ \\
\hline & & Alto & $46.4(13)$ \\
\hline & \multirow{3}{*}{ QV física } & Baixo & $28.6(8)$ \\
\hline & & Médio & $57.1(16)$ \\
\hline & & Alto & $14.3(4)$ \\
\hline & \multirow{3}{*}{ QV psicológica } & Baixo & $28.6(8)$ \\
\hline & & Médio & 46.4 (13) \\
\hline & & Alto & $25(7)$ \\
\hline & \multirow{3}{*}{ QV relações sociais } & Baixo & $50(14)$ \\
\hline & & Médio & $25(7)$ \\
\hline & & Alto & $25(7)$ \\
\hline & \multirow{3}{*}{ QV ambiente } & Baixo & $35.7(10)$ \\
\hline & & Médio & $39.3(11)$ \\
\hline & & Alto & $25(7)$ \\
\hline \multirow{3}{*}{ Auto-imagem } & \multirow{3}{*}{ Satisfação com a aparência } & Baixo & $28.6(8)$ \\
\hline & & Médio & $50(14)$ \\
\hline & & Alto & $21(6)$ \\
\hline
\end{tabular}

Fuente: elaboración propia.

Teste $t$ foi utilizado para examinar possíveis diferenças nos resultados da qualidade de vida e da auto-imagem em relação ao gênero do paciente, porém os resultados não mostraram diferenças significativas. A fim de examinar a correlação existente entre as variáveis qualidade de vida e auto-imagem, fez-se a correlação de Pearson. Observou-se na Tabela 4 que a qualidade de vida total correlacionou-se positivamente com auto-imagem, assim como suas subescalas, com exceção da subescala relações sociais. A fim de examinar o poder preditivo da auto-imagem para a qualidade de vida de pacientes com câncer de cabeça e pescoço, utilizou-se a análise de regressão linear (Tabela 5). Verificou-se que a auto-imagem foi uma variável preditora de maneira significativa da qualidade de vida total $(\beta$ $=0.664 ; \mathrm{p}<0.01$ ) explicando $44 \%$ da variância; da qualidade de vida física $(\beta=0.515 ; \mathrm{p}<0.01)$, explicando $26.5 \%$ da variância; da qualidade de vida psicológica $(\beta=0.654 ; p<0.01)$, explicando $42.8 \%$ e da qualidade de vida relacionada ao meio-ambiente $(\beta=0.630 ; \mathrm{p}<0.01)$ explicando $39.7 \%$.

\section{TABELA 4}

Correlações

\begin{tabular}{lc}
\hline & Auto-imagem Total \\
\hline Qualidade de Vida Total & $0.664(* *)$ \\
Qualidade de Vida - Físico & $0.515(* *)$ \\
Qualidade de Vida - Psicológico & $0.654(* *)$ \\
Qualidade de Vida - Relações Sociais & 0.281 \\
Qualidade de Vida - Meio Ambiente & $0.63(* *)$ \\
\hline
\end{tabular}

$\mathrm{QV}=$ qualidade de vida $* * \mathrm{p}<0.01 * \mathrm{p}<0.05$

Fuente: elaboración propia. 
TABELA 5

Análise de Regressão

\begin{tabular}{lcccc}
\hline & QV TOTAL & QV FISICA & QV PSICOLOGICO & QV MEIO \\
\hline & $\beta$ & $\beta$ & $\beta$ & $\beta$ \\
Auto-imagem & $0.664^{* *}$ & $0.515^{* *}$ & $0.654^{* * *}$ & $0.63^{* *}$ \\
$\mathrm{R}^{2}$ & $0.44^{* *}$ & $0.265^{* *}$ & $0.428^{* *}$ & $0.397^{* *}$ \\
\hline
\end{tabular}

$\mathrm{QV}=$ qualidade de vida ${ }^{*} p<0.05,{ }^{* *} p<0.01 \beta=$ coeficiente de regressão estandarizado

Fuente: elaboración propia.

\section{Discussão}

O objetivo do presente estudo foi examinar a auto-imagem e a qualidade de vida dos pacientes acometidos de câncer de cabeça e pescoço. Apesar de haver poucos estudos anteriores tratando especificamente dessas duas variáveis nesse grupo de pacientes, a expectativa inicial era de que a auto-imagem teria influência significativa na qualidade de vida desses sujeitos.

As análises descritivas e de níveis de qualidade de vida e auto-imagem mostrou que, apesar da doença e de suas possíveis deformações e limitações, a maioria desses pacientes percebiam sua qualidade de vida na média, assim como sua auto-imagem, exceção feita à qualidade de vida relações sociais, que parece ser a mais prejudicada. No mesmo sentido, a análise correlacional mostrou que os construtos qualidade de vida e auto-imagem em pacientes com câncer na face estão significativamente relacionados, com uma única exceção referindo-se à dimensão relações sociais da qualidade de vida e auto-imagem. Considerando que a face é uma parte do corpo fundamental na comunicação com o outro (Schilder, 1999), e que estudos internacionais haviam demonstrado um déficit nas relações sociais de pacientes com alterações no rosto por câncer (Babin et al., 2008; Bull \& Rumsey, 1998; Moss, 2005; Newell \& Clarke, 2000), poderíamos pensar que essas dificuldades nas relações sociais poderiam ser decorrentes da auto-imagem deformada. No entanto, a dimensão relações sociais da qualidade de vida foi a única a não se correlacionar com autoimagem, demonstrando independência entre esses dois conceitos. Mais estudos com amostras la- tinoamericanas são necessários para esclarecer essa questão, pois é possível que pelas características da cultura da região, a pessoa acometida pelo câncer de cabeça e pescoço se sinta acolhida e apoiada pela família, ao contrário de outras culturas, o que hipoteticamente poderia fazer com as alterações na sua face não resultassem em diminuição da qualidade de vida social.

A análise de regressão linear mostrou que a autoimagem é um fator preditor significativo de qualidade de vida de pacientes com câncer de cabeça e pescoço. Com base nesse resultado, pode-se afirmar que a qualidade de vida desses pacientes, com exceção da dimensão relações sociais, está intimamente ligada ao conceito de auto-imagem. Assim, é importante dar atenção especial às graves mudanças físicas que ocorrem com a doença e com o tratamento para o câncer, pois com uma piora da auto-imagem, provavelmente a qualidade de vida do indivíduo também decresce, o que tem impacto em diferentes aspectos de sua vida.

Segundo Lima (2002), é importante constatar as dimensões nas quais os pacientes apresentam menor índice de qualidade de vida e quais são os aspectos que a influenciam. Com base nos resultados do presente estudo, em primeiro lugar observa-se que as relações sociais é a dimensão da qualidade de vida mais prejudicada, e que essa não tem relação com a auto-imagem. Futuras pesquisas deverão investigar outras possíveis variáveis associadas a esse déficit na qualidade de vida social. Em contrapartida, percebe-se que a qualidade de vida psicológica é a mais afetada pela auto-imagem alterada do paciente com câncer na face. Desse modo, intervenções direcionadas ao preparo psicológico para a cirurgia 
de reconstrução da face, para o tratamento médico, assim como para a aceitação de sua nova imagem, poderão ajudar o paciente a ter uma melhor qualidade de vida dentro das limitações impostas pela sua enfermidade e inclusive a sua sobrevivência, já que o estudo de Karvonen-Gutierrez et al. (2008) verificou que a qualidade de vida é preditora do tempo de sobrevivência desses pacientes.

Os resultados desse estudo evidenciam mais uma vez que pacientes com câncer, em especial aqui aqueles que são afetados na região da face, merecem atenção psicológica já que a desfiguração numa parte tão visível e importante do corpo humano parece ser fator agravante para mudanças na sua auto-imagem. Para ajudar a melhorar a qualidade de vida desses pacientes, devemos atuar em conjunto com a equipe médica tão logo seja diagnosticado câncer na face para poder estar junto do paciente em todas as fases de evolução da doença, do tratamento cirúrgico e de sua reabilitação (Devins, Otto, Irish \& Rodin, 2010). Devemos atuar desde uma preparação pré-cirúrgica, pois é na realização da cirurgia que vai se concretizar a mutilação de face, até um acompanhamento pós-alta.

De acordo com Dropkin (1999), o apoio psicológico recebido antes da cirurgia facilita o enfrentamento do desfiguramento pós-cirúrgico bem como reduz o período de internação hospitalar. A mutilação na face em alguns casos é tão grave que alguns pacientes chegam, inclusive, a ser mantidos em quartos de isolamento para evitar um mal-estar para o paciente em ter que se confrontar com sua nova auto-imagem perante outros colegas de quarto. Nesses casos, o acompanhamento psicológico preventivo tem como objetivo detectar alguns indicadores precoces de risco e de dificuldades singulares explícitas tanto do paciente quanto de seus familiares. Igualmente é importante a equipe interdisciplinar estar atenta e ter uma boa comunicação entre seus membros.

O presente estudo proporcionou um melhor entendimento acerca da relação entre qualidade de vida e auto-imagem em pacientes com câncer na face. Porém, sugere-se para futuras pesquisas a associação do uso de instrumentos psicométricos a entrevistas a fim de examinar profundamente a experiência subjetiva desses pacientes. Também é importante ampliar a amostra para que seja possível fazer comparações entre diferentes grupos de pacientes (ex. sexo, tipo de diagnóstico, etc.) e ter grupos controle para avaliarmos as diferenças entre qualidade de vida e auto-imagem em distintos grupos clínicos.

\section{Referências}

Amar, A., Rapoport, A., Franzi, S. A., Bisordi, C. \& Lehn, C. N. (2002). Qualidade de vida e prognóstico nos carcinomas epidermóides de Cabeça e Pescoço. Revista Brasileira de Otorrinolaringologia, 69, 400-403.

Babin, E., Sigston, E., Hitier, M., Dehedin, D., Marie, J. P. \& Choussy, O. (2008). Quality of life and head and neck patients: Predictive factors, functional and psychosocial outcome. European Archives of Oto-Rhino-Laryngology, 265, 265-270.

Bull, R. \& Rumsey, N. (1998). The social psychology of facial appearance. New York: Springer-Verlag.

Callanhan, C. (2004). Facial disfigurement and sense of self in head and neck cancer. Society Work and Health Care, 40, 73-87.

Carvalho, M. M. M. J. (1998). Psico-oncologia no Brasil: Resgatando o viver. São Paulo: Summus.

Carvalho, C. A., Cunha, M. E., Giugliani, R., Bakos, L. \& Ashton-Prolla, P. (2004). Melanoma hereditário: prevalência de fatores de risco em um grupo de pacientes no sul do Brasil. Anais Brasileiros de Dermatologia, 79, 53-60.

Devins, G. M., Otto, K. J., Irish, J. C. \& Rodin, J. M. (2010). Head and neck cancer. In J. C. Holland, W. Breitbart, P. B. Jacobsen, M. S. Lederberg, M. M. Loscalzo \& R. S. McClorke (Eds.), PsychoOncology (2nd ed., pp. 135-139). New York: Oxford University Press.

Diniz, D. P. \& Schor, N. (2006). Guia de qualidade de vida (Série de guias de medicina ambulatorial e hospitalar UNIFESP-Escola Paulista de Medicina). Barueri, SP: Manole.

Dropkin, M. J. (1999). Body image and quality of life after head and neck cancer surgery. Cancer Practice, 7, 309-313. 
Ferreira, M. C. \& Leite, N. G. M. (2002). Adaptação e validação de um instrumento de avaliação da satisfação com a imagem corporal. Avaliação Psicológica, 1, 141-149.

Fleck, M., Leal, O. F., Louzada, S., Chachamovich, E., Vieira, G., Santos, L. \& Pinzon, V. (1999). Desenvolvimento da versão em português do instrumento de avaliação de qualidade de vida da OMS (WHOQOL-100). Revista Brasileira de Psiquiatria, 21, 19-28.

Fleck, M., Louzada, S., Xavier, M., Chachamovich, E., Vieira, G., Santos, L. \& Pinzon, V. (2000). Aplicação da versão em português do instrumento abreviado de avaliação da qualidade de vida "WHOQOL-brief". Revista de Saúde Pública, 34, 178-183.

Gilony, D., Bilboa, D., Blumstein, T., Murad, H., Talmi, Y. P., Kronenberg, J. \& Wolf, M. (2005). Effects of tracheostomy on well-being and body-image perceptions. Otolaryngology-Head and Neck Cancer Surgery, 133, 366-371.

Harris, D. L. \& Carr, A. T. (2001). Prevalence of concern about physical appearance in the general population. British Journal of Plastic Surgery, 54, 223-226.

Instituto Nacional do Câncer. (2010). Estatísticas do Câncer. Acesso em 25 junho, 2010, http://www1. inca.gov.br/vigilancia/incidencia.html

Karvonen-Gutierrez, C. A., Ronis, D. L., Fowler, K. E., Terrell, J. E., Gruber, S. B. \& Duffy, S. A. (2008). Quality of life scores predict survival among patients with head and neck cancer. Journal of Clinical Oncology, 26, 2754-2760.

Katz, M. R. (2000). Reliability and validity of an observer-rated disfigurement scale for head and neck cancer patients. Head $E$ Neck, 22, 132-141.

Katz, M. R., Irish, J. C., Devins, G. M., Rodin, G. M. \& Gullane, P. J. (2003). Psychosocial adjustment in head and neck cancer: The impact of disfigurement, gender and social support. Head $\mathbb{E}$ Neck, 25, 103-112.

Kowalski, L. P. (2005). Afecções cirúrgicas do Pescoço. São Paulo: Atheneu.

Lima, A. F. B. S. (2002). Qualidade de vida em pacientes do sexo masculino dependentes de álcool. Dissertação de mestrado não publicada, curso de pós-gradua- ção em Medicina, Clínica Médica, Universidade Federal do Rio Grande do Sul, Porto Alegre, Brasil.

MacGregor, F. C. (1990). Facial disfigurement: Problems and management of social interaction and implications for mental health. Aesthetic Plastic Surgery, 14, 249-257.

Moss, T. P. (2005). The relationships between objective and subjective ratings of disfigurement severity, and psychological adjustment. Body Image, 2, 151-159.

Newell, R. J. (1999). Altered body-image: A fear-avoidance model of psycho-social difficulties following disfigurement. Journal of Advanced Nursing, 30, 1230-1238.

Newell, R. J. \& Clarke, M. (2000). Evaluation of self-help leaflet in treatment of social difficulties following facial disfigurement. International Journal of Nursing Studies, 37, 381-388.

Nora, A. B., Panarotto, D., Lovatto, L. \& Boniatti, C. (2004). Freqüência de aconselhamento para prevenção de câncer de pele entre as diversas especialidades médicas em Caxias de Sul. Anais Brasileiro Dermatologia, 79, 45-51.

Oliveira, R. A. (2004). O sujeito e o corpo perante a incapacidade física. Revista Portuguesa de Psicossomática, 6, 63-67.

Pedrolo, F. T. \& Zago, M. M. F. (2000). A imagem corporal alterada do laringectomizado: Resignação com a condição. Revista Brasileira de Cancerologia, 46, 407-415.

Price, B. (1990). Body image: Nursing concepts and care. Great Britain: Prentice Hall.

Remor, E. (2005). Desarrollo de uma medida específica para la evaluación de la calidad de vida em pacientes adultos viviendo com hemofilia em America Latina: el Hemolatin-Qol. Revista Interamericana de Psicologia, 39, 211-220.

Rogers, S. M., Ahad, S. A. \& Murphy, A. P. (2007). A structured review and them analysis of papers published on quality of life in head and neck cancer: 2000-2005. Oral Oncology, 43, 843-868.

Rumsey, N., Clarcke, A. White, P., Wyn- Williams, M. \& Garlick, W. (2004). Altered body image: appearance-related concerns of people with visible disfigurement. Journal of Advanced Nursing, 48, 443-453. 
Schilder, P. (1999). A imagem do corpo: As energias construtivas da psique. São Paulo: Martins Fontes.

Seidl, E. M. F. \& Zannon, C. M. L. C. (2004). Qualidade de vida e saúde: aspectos conceituais e metodológicos. Caderno de Saúde Pública, 20, 580-588.

Sociedade Brasileira De Dermatologia. (2006). Data analysis of the Brazilian Society of Dermatology skin cancer prevention campaign, 1999 to 2005. Anais Brasileiros de Dermatologia, 81, 533-539.

Vickery, L. E., Latchford, G. Hewison, J., Bellew, M. $\&$ Feber, T. (2003). The impact of head and neck cancer and facial disfigurement on the quality of life of patients and their partners. Head $\mathcal{E}$ Neck, 25, 289-296.

Vidrio, R. M. G. \& Lozano, N. C. (2007). Confrontando al melanoma em el siglo XXI. Medicina Cutânea Ibero-Latino-Americana, 35, 3-13.

World Health Organization. (2010). Programme on Mental Health. Acesso em 22 de junho de 2010, em http://www.who.int/mental_health/media/68.pdf 
